We report a rare case of esophageal bezoar, thought to have formed after oral intake of sucralfate. A 62-year-old man, who was wheelchair-bound due to paralysis of both lower limbs following surgery for a spinal tumor, and with a history of partial gastrectomy for gastric ulcer, was diagnosed with cancer of the pancreatic head. The patient refused any treatment apart from symptomatic care.

The patient was admitted to hospital after sudden dysphagia and severe vomiting. The X-ray examination showed no appearance suspicious for ileus. The upper gastrointestinal series showed an obstruction in the lower part of the esophagus, but no stenosis of the duodenum was shown upon examination using contrast medium which seeped through the obstruction later. Chest computed tomography (CT) showed an air-containing foreign body in the esophageal lumen, dilating the oral side of the esophagus (Figure 1). Endoscopy showed a cream-colored soft bezoar that was fixed in the lower part of the esophagus, causing an obstruction (Figure 2 ). It was broken into pieces endoscopically and drawn down into the stomach. An ulcer was observed in the esophageal mucosa where the bezoar had been trapped.

Part of the the bezoar was analyzed and found to contain a large amount of aluminum. No bezoar had been detected during previous endoscopic examination prior to the onset of treatment with sucralfate for remnant gastritis and esophagitis. The bezoar was therefore considered to have formed from the sucralfate, which has a concentration of aluminum that is not found in nature.

Two factors are considered to be related to esophageal bezoar formation (not including gastric bezoar that has migrated to the esophagus): esophageal disorders causing peristaltic abnormality, and the ingestion of material that is easily aggregated. The former has been reported in Zenker's diverticulum [1], achalasia [2],

\title{
Sucralfate-formed esophageal bezoar detected following sudden vomiting
}

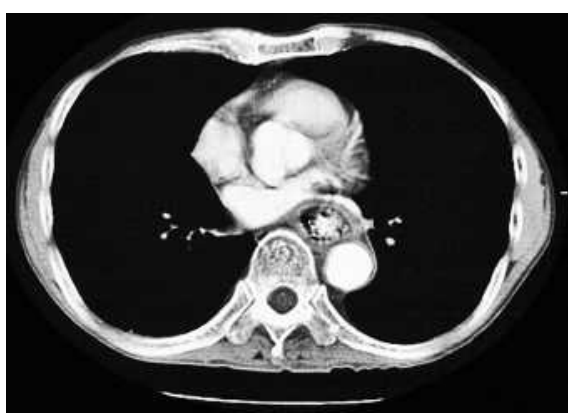

Figure 1 An air-containing bezoar in the esophageal lumen is located by computed tomography (CT).
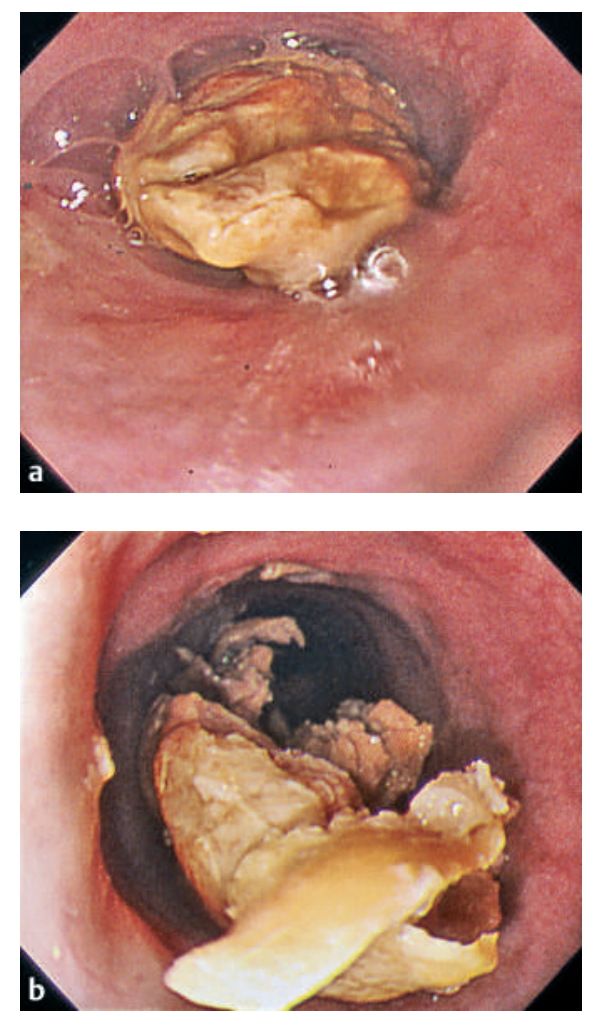

Figure 2 a Endoscopic examination reveals a cream-colored bezoar blocking the esophageal lumen. b The bezoar was an agglomeration of muddy material, easily destroyed by biopsy forceps.

systemic sclerosis [3], and post-radiotherapy, and the latter has been reported following oral intake of granular laxative [4], and sucralfate as in the present case. Most bezoars are treated by endoscopic destruction or removal. Most cases occur in individuals with retarded peristalsis due to a bed-ridden state and enteral feeding.

Endoscopy_UCTN_Code_CCL_1AB_2AF

Y. Kise ${ }^{1,2}$, R. Aihara², O. Chino',

S. Yamamoto' ${ }^{1}$, T. Hara', T. Nishi',

T. Kenmochi', H. Shimada ${ }^{1}$,

H. Makuuchi ${ }^{1}$

${ }^{1}$ Department of Surgery, Tokai University School of Medicine, Bohseidai Isehara, Japan

2 Department of Surgery, Ozawa Hospital, Kanagawa, Japan.

\section{References}

${ }^{1}$ Tsai CJ. Bezoar in a Zenker's diverticulum. Am J Gastroenterol 1994; 89: 944 - 946

2 Shah SW, Khan AA, Alam A et al. Esophageal bezoar in achalasia: a rare condition. J Clin Gastroenterol 1997; 25: 395-396

${ }^{3}$ Grosskopf I, Streifler J, Garty M, Rosenfeld JB. Esophageal bezoar in progressive systemic sclerosis. J Clin Gastroenterol 1986; 8: 695 696

${ }^{4}$ Brown DC, Doughty JC, George WD. Surgical treatment of oesophageal obstruction after ingestion of a granular laxative. Postgrad Med J 1999; 75: 106

\section{Corresponding Author}

\section{Y. Kise}

Department of Surgery

Tokai University School of Medicine Bohseidai

Isehara

Japan 259-1193

Fax: $\quad+81-463-956491$

E-mail: ykisejp@ybb.ne.jp

DOI: $10.1055 / s-2006-944712$ 